\title{
C-Peptide Area Under the Curve at Glucagon Stimulation Test Predicts Glucose Improvements by GLP-1 Receptor Analogue: A Retrospective Observational Study
}

\author{
Azusa Ohbatake $\cdot$ Kunimasa Yagi - Shigehiro Karashima - Yuki Shima $\cdot$ Yukiko Miyamoto • \\ Hiroyuki Asaka · Satoko Okazaki · Mitsuhiro Kometani · Masa-aki Kawashiri · Yoshiyu Takeda • \\ Takashi Yoneda $\cdot$ Daisuke Chujo (i)
}

Received: December 29, 2018 / Published online: February 20, 2019

(C) The Author(s) 2019

\section{ABSTRACT}

Introduction: Despite the widespread use of glucagon-like peptide-1 (GLP-1) receptor agonists (GLP-1ras) to improve glycemic regulation, with a low risk of hypoglycemia and weight reduction, their effectiveness varies among individuals. This study aimed to identify predictors of the efficacy of GLP-1ra on Hemoglobin A1c (HbA1c) in patients with insulinindependent diabetes.

Methods: In total, 58 patients with insulin-independent diabetes were included. Patients

Azusa Ohbatake and Shigehiro Karashima contributed equally to the work.

Enhanced Digital Features To view enhanced digital features for this article go to https://doi.org/10.6084/ m9.figshare.7695638

A. Ohbatake $\cdot$ S. Karashima $\cdot$ Y. Shima .

Y. Miyamoto $\cdot$ H. Asaka $\cdot$ S. Okazaki · M. Kometani

- M. Kawashiri · Y. Takeda · T. Yoneda - D. Chujo

Department of Internal Medicine, Kanazawa

University Graduate School of Medical Science,

Kanazawa, Japan

K. Yagi

First Department of Internal Medicine, University of Toyama, Toyama, Japan

T. Yoneda

Department of Health Promotion and Medicine of the Future, Kanazawa University Graduate School of Medical Science, Kanazawa, Japan were included if their $\beta$-cell function was evaluated via a glucagon stimulation test (GST) before the introduction of GLP-1ra therapy. $\beta$ Cell function-related indices, such as the C-peptide index (CPI), increments in C-peptide immunoreactivity (CPR) after glucagon stimulation $(\triangle \mathrm{CPR})$, and the area under the CPR curve (CPR-AUC) during the GST, were evaluated. HbA1c and body weight (BW) were measured at 6 and 12 months after the initiation of GLP-1ra. Results: A univariate regression analysis revealed a significant correlation between CPRAUC and changes in HbA1c at 6 months and with changes in BW at 6 and 12 months. A multivariate regression analysis revealed that CPR-AUC was significantly correlated with changes in HbA1c at 6 months. A receiver-operating characteristic analysis revealed that 21.9 $\mathrm{ng} / \mathrm{ml} \cdot \mathrm{min}$ CPR-AUC was the optimal cut-off value to predict an HbA1c level $<7 \%$, i.e., 53 $\mathrm{mmol} / \mathrm{mol}$.

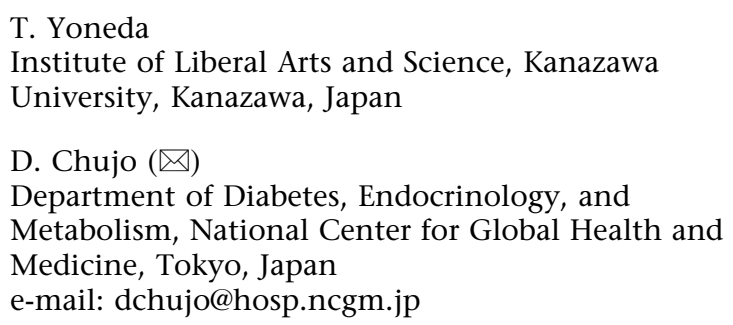


Conclusion: Residual $\beta$-cell function, as assessed via CPR-AUC in the GST, is an effective predictor of the efficacy of GLP-1ras.

Keywords: C-peptide; GLP-1 receptor agonist; Glucagon stimulation test; Insulin-independent diabetes; Liraglutide; Lixisenatide

\section{INTRODUCTION}

Type 2 diabetes is characterized by hyperglycemia and is associated with a high risk of cardiovascular, microvascular, and other complications; it is a heterogeneous disease involving pancreatic $\beta$-cell dysfunction and insulin resistance $[1,2]$. A progressive decline in $\beta$-cell function decreases insulin secretion and disrupts glucose homeostasis [3-5].

Glucagon-like peptide-1 (GLP-1), a peptide produced in the entero-endocrine L-intestinal cells, is a major incretin hormone that stimulates insulin secretion after nutrient ingestion. Owing to its involvement in pancreatic $\beta$-cell proliferation and survival and the regulation of glucagon secretion, food intake, and gastric and intestinal motility, it has helped develop efficient pharmacologic treatments for diabetes and obesity $[6,7]$.

Several GLP-1 receptor agonists (GLP-1ras) with distinct structural and pharmacokinetic properties are currently used to treat type 2 diabetes and to prevent cardiovascular disease, the main cause of death in patients with type 2 diabetes. These agonists improve glycemic regulation with a low risk of hypoglycemia and weight reduction. Furthermore, recent trials on cardiovascular outcomes have reported that GLP-1ras liraglutide and semaglutide reduce the occurrence rate of cardiovascular events and deaths among patients with type 2 diabetes at high cardiovascular risk $[8,9]$.

However, some clinical trials have reported that the glucose-lowering effect of GLP-1ras potentially differs among individuals, with some patients exhibiting insufficient responses in the clinical setting. Accordingly, predictors of the therapeutic response to GLP-1ra are needed to identify patient subsets potentially benefiting from GLP-1ras treatment. We hypothesized that residual insulin secretory capacity in patients with type 2 diabetes is an important predictor of the glucose-lowering effect of GLP-1ra. Hence, the present study aimed to identify factors predicting the efficacy of GLP1ras on Hemoglobin $\mathrm{A} 1 \mathrm{c}(\mathrm{HbA1c})$ in patients with insulin-independent diabetes. Furthermore, since GLP1-ra promotes weight loss, along with glucose-lowering effects, we evaluated the association between residual $\beta$-cell function and weight loss effects of GLP-1ra.

\section{METHODS}

\section{Study Design and Ethics}

This retrospective, single-center study was conducted at Kanazawa University Hospital between November 2010 and June 2014. All procedures were performed in accordance with the 1964 Helsinki Declaration and its later amendments. The study was approved by the ethics committees at the University of Kanazawa (no. 2014124), and patient consent was waived by the ethics committee owing to the retrospective nature of the study. Since this study was a retrospective observational study that used non-identifiable data obtained by the treating physicians, the study was not registered in the national database of clinical trials in accordance with the ethics committee's decision. Furthermore, the study included an optout method, as shown on our hospital website and the poster at Kanazawa University Hospital, and subjects could opt out of the study at any time.

\section{Participants and Study Assessment}

The medical records of patients with insulinindependent diabetes who initially received GLP-1ra therapy at Kanazawa University Hospital between November 2010 and June 2014 were reviewed. Data were collected $24 \pm 2$ or $48 \pm 2$ weeks after GLP-1ra administration as the data at 6 and 12 months. The exclusion criteria were renal failure [estimated glomerular filtration rate $(\mathrm{eGFR})<30 \mathrm{ml} / \mathrm{min} / 1.73 \mathrm{~m}^{2}$ ], 
severe hepatic dysfunction, pregnancy or possible pregnancy, hypersensitivity to the trial drugs, or a change in oral hypoglycemic agents (OHA) within 3 months. Patients with insulindependent diabetes with obliterated $\beta$-cell function were also excluded. Insulin dependency was defined by a combination of fasting C-peptide immunoreactivity $(\mathrm{CPR}) \leq 0.5 \mathrm{ng} / \mathrm{ml}$ and stimulated CPR $\leq 1.0 \mathrm{ng} / \mathrm{ml}$ by glucagon. Finally, the patients were primarily administered the following treatments before switching to GLP-1ra: insulin therapy ( $n=38$, daily insulin injection $0.36 \pm 0.25 \mathrm{IU} / \mathrm{day} / \mathrm{kg}$ ) or glimepiride ( $n=1,0.5 \mathrm{mg}$ daily). Additionally, 19 patients were administered GLP-1ra therapy without a switch from the previous therapy. The patients were continuously advised on lifestyle modifications, including exercise and diet, as usual.

HbA1c, body weight (BW), blood pressure (BP), eGFR, and urinary albumin excretion (UAE) were measured at 6 and 12 months after GLP-1ra administration. The duration of diabetes was defined in accordance with the criteria of the Japan Diabetes Society as years after diagnosis of the disease. HbA1c, eGFR, and UAE were determined through standard laboratory procedures. After the switch to or addition of GLP-1ra, the dosages of GLP-1ra and additional OHA were adjusted to achieve the target HbA1c level $(<7.0 \%, 53 \mathrm{mmol} / \mathrm{mol})$. The patients continuously received GLP-1ra treatment for 6 months and were administered sulfonylureas during 6 months at the following doses: glimepiride $(n=23) 0.84 \pm 0.83 \mathrm{mg}$ and gliclazide $(n=1) 10 \mathrm{mg}$.

\section{Endogenous Insulin Secretion from $\beta$-Cells}

Pancreatic $\beta$-cell function was evaluated via a glucagon stimulation test (GST) before the initiation of GLP-1ra therapy. In the GST, stimulation was carried out through i.v. injection of $1 \mathrm{mg}$ of glucagon (Glucagon G Novo; Novo Nordisk, Bagsvaerd, Denmark). The levels of fasting plasma glucose (FPG), plasma glucose 6 min after glucagon stimulation, fasting serum C-peptide (CPRO), and serum C-peptide $6 \mathrm{~min}$ after glucagon stimulation (CPR6) were measured. Serum C-peptide levels were measured using an Immunoenzymometric Assay Kit (TOSOH, Tokyo, Japan). Several parameters associated with endogenous insulin secretion, such as the C-peptide index (CPI), increments of CPR after glucagon stimulation $(\triangle \mathrm{CPR})$, and the area under the CPR curve during the GST (CPR-AUC), were obtained. CPI, $\triangle \mathrm{CPR}$, and CPR-AUC were calculated as follows: CPI, CPR0 $\times 100 /$ FPG $[10,11] ; \Delta$ CPR $(\mathrm{ng} / \mathrm{ml})$, CPR6 - CPR0 [12]; CPR-AUC, (CPR0 + CPR6) $\times 6 / 2$ [13].

\section{Statistical Analysis}

Data are expressed as mean \pm SD values and analyzed using commercially available statistical software (JMP version 11, SAS Institute Inc., Cary, NC, USA); $P<0.05$ was considered statistically significant. The differences between baseline and follow-up measurements were analyzed using paired $t$ tests. The associations between parameter changes and biomarkers of $\beta$-cell function before administration were examined via Pearson correlation coefficient analysis. For variables with significant correlations, a multiple linear regression analysis was performed to identify independent factors, adjusting for age, sex, body mass index (BMI), disease duration, and baseline HbA1c. The receiver-operating characteristic (ROC) curve was used to assess the appropriate cut-off values for successful introduction of GIP-1ra. Additionally, the sensitivity and specificity for the optimal cut-off value were calculated from the areas under the curve (AUC).

\section{RESULTS}

\section{Clinical Characteristics}

Fifty-eight patients (42 males, aged $63 \pm 13$ years) with insulin-independent diabetes were included in the study cohort. The duration of diabetes was $18 \pm 10$ years. The FPG was $146 \pm 29 \mathrm{mg} / \mathrm{dl}$; fasting CPR, $1.9 \pm 0.8 \mathrm{ng} /$ $\mathrm{ml}$ before GLP-1ra administration; CPI, $\triangle \mathrm{CPR}$, and CPR-AUC values at baseline, $1.3 \pm 0.6$, 
$2.0 \pm 1.3 \mathrm{ng} / \mathrm{ml}$, and $17.3 \pm 7.7 \mathrm{ng} / \mathrm{ml} \mathrm{min}$, respectively. Fifty-two patients were administered liraglutide, and six patients were administered lixisenatide. Of these patients, three discontinued GLP-1ra (hyperglycemia $n=1$, nausea $\mathrm{n}=1$, refusing GLP-1ra injection therapy after switching $n=1$ ). Cases of severe hypoglycemia or diabetic ketoacidosis were not reported. Table 1 shows the changes in parameter values for patients with insulin-independent diabetes treated with GLP-1ra.

\section{Correlation Between CPR-AUC and HbA1c Reduction}

As summarized in Table 1, treatment with GLP1ra for 6 and 12 months did not significantly reduce $\mathrm{HbA1c}$, with changes from $7.8 \pm 1.4 \%$, $62 \pm 15.5 \mathrm{mmol} / \mathrm{mol}$ to $7.4 \pm 1.0 \%, \quad 57 \pm$ $10.7 \mathrm{mmol} / \mathrm{mol} \quad(P=0.112)$ and $7.4 \pm 1.0 \%$, $57 \pm 11.1 \mathrm{mmol} / \mathrm{mol}(P=0.517)$, respectively.

A univariate regression analysis revealed a significant correlation between CPR-AUC and changes in HbA1c at 6 months $\left(R^{2}=0.081\right.$, $P=0.048)$; however, this correlation was not significant at 12 months $\left(R^{2}=0.052, P=0.146\right)$ (Fig. 1a, b). Furthermore, CPR-AUC was significantly correlated with changes in HbA1c for 6 months in accordance with the multivariate regression analysis after adjusting for age, sex, BMI, disease duration, and baseline HbA1c $(\beta=-0.415, P<0.001)$ (Table 2$)$.

CPI and $\triangle \mathrm{CPR}$ were not significantly correlated with changes in HbA1c at 6 and
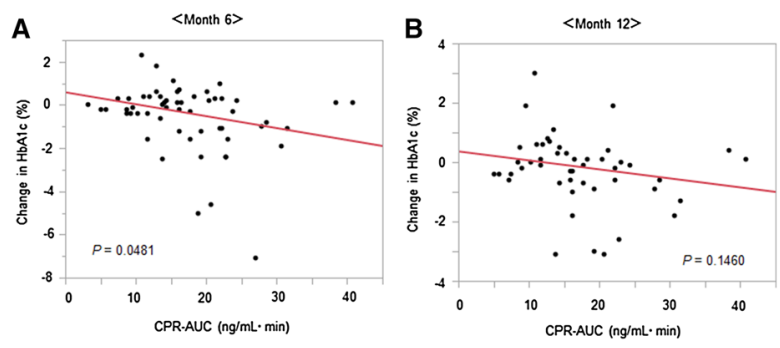

Fig. 1 Correlations between CPR-AUC and changes in HbAlc at 6 months (a) and 12 months (b) after GLP-1ra administration. CPR C-peptide immunoreactivity, CPR$A U C$ area under the CPR curve during the glucagon stimulation test, GLP-1ra glucagon-like peptide-1 receptor agonist

12 months (CPI: $R^{2}=0.033, P=0.212$ and $\triangle$ CPR: $R^{2}=0.074, P=0.059$ against the change in HbA1c at 6 months, CPI: $R^{2}=0.008$, $P=0.576$ and $\triangle \mathrm{CPR}: R^{2}=0.043, \quad P=0.189$ against the change in HbA1c at 12 months).

To determine the cut-off values for achieving an HbA1c level of $<7.0 \%, 53 \mathrm{mmol} / \mathrm{mol}$ at 6 months, ROC analyses were performed for CPR-AUC. The estimated cut-off point was 21.9 with $62 \%$ sensitivity and $89 \%$ specificity (Fig. 2). The cut-off points for CPI and $\triangle \mathrm{CPR}$ were 1.29 (AUC 0.771 with $85 \%$ sensitivity and $68 \%$ specificity) and 1.8 (AUC 0.795 with $85 \%$ sensitivity and $59 \%$ specificity).

The subjects were segregated into two groups in accordance with the cut-off value for each parameter $(\mathrm{CPI}=1.29, \Delta \mathrm{CPR}=1.8$, and CPR$A U C=21.9)$. Figure 3 shows the changes in

Table 1 Clinical parameters before and after GLP-1ras introduction

\begin{tabular}{lccc}
\hline Characteristics & Baseline & 6 months & 12 months \\
\hline BW $(\mathrm{kg})$ & $71.0 \pm 19.6$ & $66.0 \pm 18.6^{* *}$ & $66.6 \pm 18.3^{* *}$ \\
HbAlc $(\%, \mathrm{mmol} / \mathrm{mol})$ & $7.8 \pm 1.4,62 \pm 15.5$ & $7.4 \pm 1.0,57 \pm 10.7$ & $7.4 \pm 1.0,57 \pm 11.1$ \\
Systolic BP $(\mathrm{mmHg})$ & $123 \pm 18$ & $124 \pm 17$ & $125 \pm 13$ \\
Diastolic BP $(\mathrm{mmHg})$ & $73 \pm 12$ & $64 \pm 9^{* *}$ & $65 \pm 10^{*}$ \\
eGFR $\left(\mathrm{ml} / \mathrm{min} / 1.73 \mathrm{~m}^{2}\right)$ & $78.1 \pm 22.2$ & $79.2 \pm 23.8$ & $81.6 \pm 19.8$ \\
UAE $(\mathrm{mg} / \mathrm{gCr})$ & $34.6 \pm 78.2$ & $57.3 \pm 85.5$ & $61.8 \pm 103.3$ \\
\hline
\end{tabular}

Data are presented as mean $\pm \mathrm{SD}$

$B W$ body weight, $B P$ blood pressure, $e G F R$ estimated glomerular filtration rate, $U A E$ urinary albumin excretion ${ }^{*} P<0.01$ and ${ }^{* *} P<0.001$ in paired $t$ tests 
Table 2 Multivariate logistic regression analysis of the CPR-AUC and changes in HbAlc

\begin{tabular}{lllll}
\hline Explanatory variable & $\boldsymbol{\beta}$ & SE & 95\% CI & \multicolumn{1}{c}{ value } \\
\hline CPR-AUC & -0.42 & 0.02 & -0.12 to -0.04 & $0.0001^{*}$ \\
Age & 0.10 & 0.01 & -0.01 to 0.04 & 0.32 \\
Sex (female) & 0.12 & 0.16 & -0.12 to 0.52 & 0.21 \\
BMI & 0.25 & 0.03 & 0.01 to 0.12 & $0.02^{*}$ \\
Disease duration & -0.01 & 0.01 & -0.03 to 0.03 & 0.89 \\
HbAlc & -0.77 & 0.09 & -0.98 to -0.62 & $<0.0001^{*}$ \\
\hline
\end{tabular}

$S E$ standard error, $C I$ confidence interval, $C P R$ C-peptide immunoreactivity, $C P R-A U C$ area under the CPR curve during the glucagon stimulation test, $B M I$ body mass index

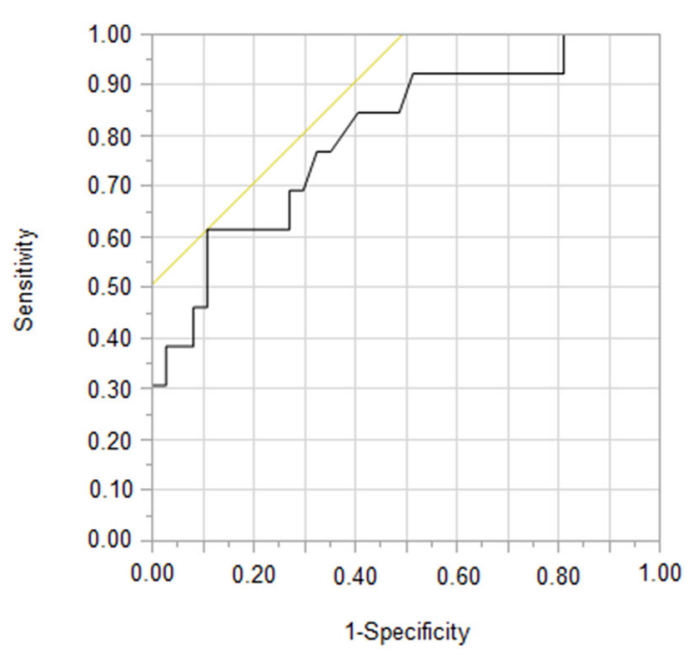

\begin{tabular}{|c|c|c|c|}
\hline & CPR-AUC & CPI & $\Delta$ CPR \\
\hline AUC & 0.800 & 0.771 & 0.795 \\
\hline $95 \% \mathrm{Cl}$ & $0.613-0.910$ & $0.616-0.904$ & $0.600-0.885$ \\
\hline Cut-off point & 21.9 & 1.29 & 1.8 \\
\hline Sensitivity (\%) & 62 & 85 & 85 \\
\hline Specificity (\%) & 89 & 68 & 59 \\
\hline
\end{tabular}

Fig. 2 ROC curves of CPR-AUC to predict HbAlc values $<7 \%, 53 \mathrm{mmol} / \mathrm{mol}$ at 6 months. CPR C-peptide immunoreactivity, $C P R-A U C$ area under the CPR curve during the glucagon stimulation test, $C P I \mathrm{C}$-peptide index, $\triangle C P R$ increments of $\mathrm{CPR}$ after glucagon stimulation, $R O C$ receiver-operating characteristic, $A U C$ area under the ROC curve, $C I$ confidence interval

HbA1c at 6 or 12 months after switching to or on addition of GLP-1ra to the treatment regimens of patients with CPR-AUC $>21.9 \quad(n=10)$ and those with CPR-AUC $\leq 21.9(n=39)$. Changes in HbA1c at 6 months differed significantly

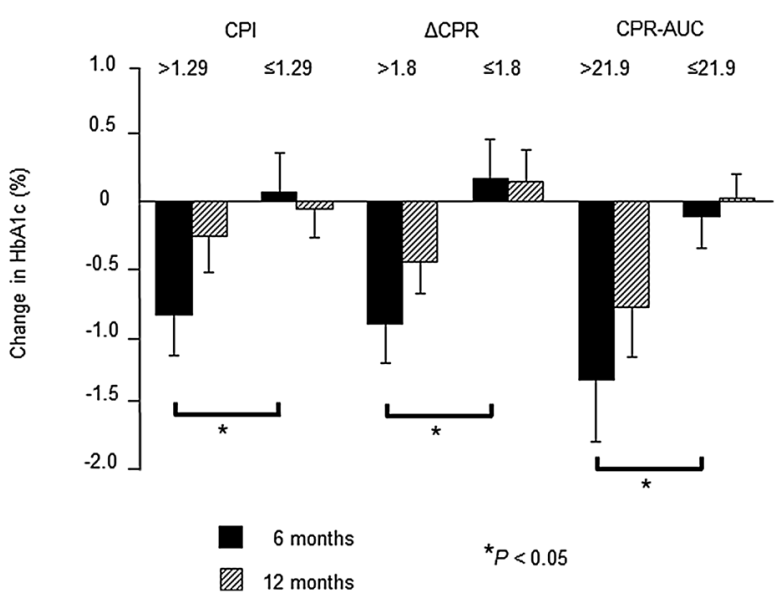

Fig. 3 Change in HbAlc during 6 or 12 months in two groups divided by each cut-off value (CPI cut-off value, 1.29; $\triangle$ CPR cut-off value, $1.8 \mathrm{ng} / \mathrm{ml}$; CPR-AUC cut-off value, $21.9 \mathrm{ng} / \mathrm{ml} \mathrm{min})$. HbAlc changes at 6 months were significantly different between groups, but differences between groups at 12 months were not statistically significant. ${ }^{*} P<0.05$ in paired $t$ tests. CPI C-peptide index, $C P R$ C-peptide immunoreactivity, $\triangle C P R$ increments of CPR after glucagon stimulation, $C P R-A U C$ area under the $\mathrm{CPR}$ curve during the glucagon stimulation test

between the two groups (CPR-AUC $>21.9$, $-1.3 \pm 2.2 \%,-14 \pm 2.2 \mathrm{mmol} / \mathrm{mol}$, and CPR$\mathrm{AUC} \leq 21.9,-0.1 \pm 1.2 \%,-1.1 \pm 2.5 \mathrm{mmol} /$ mol for 6 months $(P=0.024))$; however, they did not differ significantly between the groups at 12 months [CPR-AUC $>21.9,-0.8 \pm 1.0 \%$, $-8.3 \pm 4.0 \mathrm{mmol} / \mathrm{mol}$ and CPR-AUC $\leq 21.9$, $0.0 \pm 1.0 \%, 0.2 \pm 2.0 \mathrm{mmol} / \mathrm{mol}$ at 12 months $(P=0.064)]$. Both CPI and $\triangle \mathrm{CPR}$ differed 
significantly between the two groups divided by the cut-off value at 6 months [CPI $>1.29, n=23$; $-0.8 \pm 2.0 \%$ and CPI $\leq 1.29, n=26 ; 0.1 \pm 0.7 \%$ at 6 months $(P=0.038)$ and $\Delta \mathrm{CPR}>1.8, n=24$; $-0.9 \pm 1.9 \%$, and $\Delta \mathrm{CPR} \leq 1.8, n=25 ; 0.2 \pm$ $0.7 \%$ at 6 months $(P=0.013)]$; however, no significant difference was observed at 12 months [CPI $>1.29,-0.3 \pm 1.5 \%$ and $\mathrm{CPI} \leq 1.29$, - $0.1 \pm 0.7 \%$ at 12 months $(P=0.563)$ and $\Delta \mathrm{CPR}>1.8,-0.4 \pm 1.2 \%$ and $\Delta \mathrm{CPR} \leq 1.8$, $0.2 \pm 0.9 \%$ at 12 months $(P=0.079)]$.

\section{Correlation Between CPR-AUC and BW Reduction}

Treatment with GLP-1ra significantly reduced BW from $70.7 \pm 20.0 \mathrm{~kg}$ to $65.9 \pm 18.8 \mathrm{~kg}$ $(P<0.0001)$ at 6 months and $66.6 \pm 18.3 \mathrm{~kg}$ $(P<0.0001)$ at 12 months (Table 1$)$.

Changes in BW at 6 and 12 months were significantly correlated with CPR-AUC $\left(R^{2}=\right.$ $0.145, P=0.010$ and $R^{2}=0.155, P=0.029$, respectively) (Fig. 4a, b). However, a multivariate regression analysis revealed that CPR-AUC was not a significant predictor of BW loss (Table 3). CPI was significantly correlated with changes in BW at 6 and 12 months $\left(R^{2}=0.142\right.$, $P=0.011$ and $R^{2}=0.163, P=0.024$, respectively); however, $\triangle \mathrm{CPR}$ was not significant $\left(R^{2}=0.065, \quad P=0.092\right.$ at 6 months and $R^{2}=0.043, P=0.261$ at 12 months).

\section{DISCUSSION}
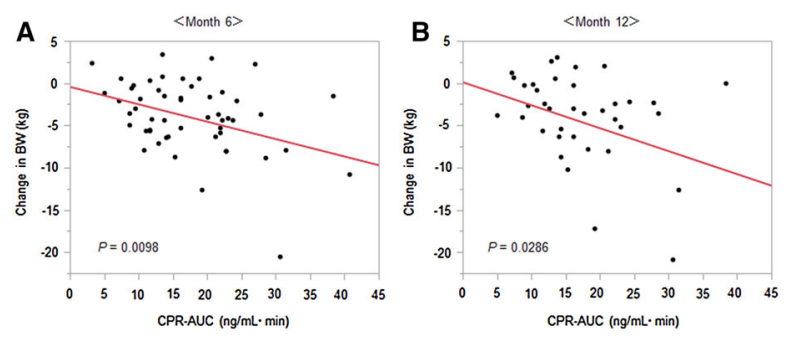

Fig. 4 Correlations between CPR-AUC and change in BW at 6 months (a) and 12 months (b) after GLP-1ra administration. CPR C-peptide immunoreactivity, CPR$A U C$ area under the $\mathrm{CPR}$ curve during the glucagon stimulation test, GLP-1ra glucagon-like peptide-1 receptor agonist, $B W$ body weight
The present results show that $\beta$-cell function should be preserved for effective GLP-1ra introduction in patients with diabetes. Changes in $\mathrm{HbA1c}$ were significantly correlated with CPR-AUC, as determined via the GST and on a multivariate regression. CPR-AUC from the GST may better predict the glucose-lowering effect of GLP-1ra than CPI or $\triangle \mathrm{CPR}$.

The GST is widely used to evaluate endogenous insulin secretion with high clinical reproducibility. CPR-AUC derived from the GST is strongly correlated with CPI and $\triangle \mathrm{CPR}$ in the GST [14]. CPI and $\triangle \mathrm{CPR}$ are reportedly useful predictors of the effectiveness of GLP-1ra [15-17]. Kondo et al. reported that CPI and stimulated CPI (CPR6 $\times 100 /$ plasma glucose at 6 min after glucagon stimulation) are candidate predictors of the efficacy of liraglutide for achieving $\mathrm{HbA} 1 \mathrm{c}<7.0 \%, 53 \mathrm{mmol} / \mathrm{mol}$ over 24 weeks. An ROC analysis revealed that the independent cut-off value for effective liraglutide introduction is 0.72 for CPI (AUC 0.75, with $81 \%$ sensitivity and $61 \%$ specificity) and 1.92 for stimulated CPI (AUC 0.76, with 53\% sensitivity and $88 \%$ specificity) [15]. Moreover, Usui et al. reported a cut-off value of 1.34 for $\Delta \mathrm{CPR}$ for the insulin-to-liraglutide switch with liraglutide termination owing to hyperglycemia for 12 weeks (AUC 0.81, with 59\% sensitivity and 95\% specificity) [16]. However, CPI and $\triangle \mathrm{CPR}$ herein were not correlated with changes in HbA1c. Differences in the results may be explained through differences in study designs and patient characteristics. The present study cohort exhibited a longer diabetes duration than those in other studies. Kozawa et al. reported that diabetes duration was significantly shorter in liraglutide responders than in liraglutide non-responders. Their ROC analyses for predicting the efficacy of liraglutide aand duration had high AUCs, and its cut-off value, 19.5 years, showed high positive predictive values and high positive likelihood ratios [18]. Furthermore, Kawata et al. reported that a shorter diabetes duration was significantly associated with liraglutide continuation after a switch from insulin therapy [19]. Because of the long diabetes duration, our patients might be weaker GLP-1ra responders than those in previous studies. Furthermore, CPI and $\triangle \mathrm{CPR}$ are 
Table 3 Multivariate logistic regression analysis of the CPR-AUC and changes in BW

\begin{tabular}{lllll}
\hline Explanatory variable & $\boldsymbol{\beta}$ & SE & $\mathbf{9 5 \%}$ CI & $\boldsymbol{P}$ value \\
\hline CPR-AUC & -0.08 & 0.08 & -0.21 to 0.13 & 0.63 \\
Age & -0.01 & 0.05 & -0.11 to 0.11 & 0.97 \\
Sex (female) & 0.00 & 0.79 & -1.58 to 1.61 & 0.99 \\
BMI & -0.55 & 0.12 & -0.62 to -0.14 & $0.003^{*}$ \\
Disease duration & -0.09 & 0.07 & -0.20 to 0.11 & 0.55 \\
HbAlc & 0.16 & 0.38 & -0.31 to 1.25 & 0.23 \\
\hline
\end{tabular}

$S E$ standard error, $C I$ confidence interval, $C P R$ C-peptide immunoreactivity, $C P R-A U C$ area under the CPR curve during the glucagon stimulation test, $B M I$ body mass index

speculated to primarily reflect basal and stimulated insulin secretion, respectively. CPR-AUC is calculated for CPR0 and CPR6 per the trapezoidal rule and may reflect total pancreatic $\beta$-cell function, including basal and stimulated insulin secretion. An ROC analysis revealed that a CPR-AUC cut-off value of 21.9 is effective for achieving HbA1c $<7 \%, 53 \mathrm{mmol} / \mathrm{mol}$. This cutoff value could be used to select potential GLP1ra responders.

GLP1-ra has not only glucose-lowering effects, but also weight loss effects via appetite suppression. CPR-AUC may also predict weight loss effects. The present univariate regression analysis revealed a significant negative correlation between CPR-AUC and changes in BW (Fig. 4); however, this correlation was not significant on multivariate regression analysis. Hence, GLP-1ra reduces body weight independent of $\beta$-cell function, concurrent with the finding that residual $\beta$-cell function does not affect weight loss and GLP-1ra reduces appetite and BW independent of $\beta$-cell function [17]. Kawata et al. reported that $\Delta \mathrm{BW}$ at 24 weeks of liraglutide administration was higher with higher baseline daily insulin doses and a longer diabetes duration [19]. Herein, baseline daily insulin doses were similar to those reported previously, while the diabetes duration was longer than those reported previously. A marked BW reduction observed herein is potentially associated with diabetes duration.

The study had several limitations. First, this retrospective, single-center study had a limited sample size; hence, prospective, multicenter studies with an adequate sample size are necessary to confirm the current findings. Second, the insulinogenic index (I.I.) in a 75-g OGTT, HOMA- $\beta$, postprandial C-peptide levels at $60 \mathrm{~min}$ [20], and urine C-peptide were not measured herein as parameters associated with the insulin secretion capacity. In particular, I.I. and urine C-peptide are useful parameters for predicting the efficacy of liraglutide in patients with type 2 diabetes [18]. Herein, the medical records of outpatients treated with insulin therapy were collected, and 24-h urine collection could not be performed; furthermore, HOMA- $\beta$ and I.I. were not measured owing to a cross-reaction with injected exogenous insulin. A 24-h urine collection, 75-g OGTT, or substitute meal test is time-consuming and generally performed during hospitalization, whereas GST is simple and easy, requiring $<10 \mathrm{~min}$, and can be performed in an outpatient clinic. The assessment of the endogenous insulin-secreting capacity via the GST is an efficient method to predict the efficacy of GLP-1ra. Third, we assessed patients receiving liraglutide or lixisenatide, dichotomized in accordance with the relative half-lives of the drugs: liraglutide is long-acting GLP-1ra, while lixisenatide is shortacting GLP-1ras. Differences in the half-lives of these two classes apparently affect their mechanistic exertion of glucose-lowering effects [21]. Several studies reported the association of residual $\beta$-cell function and the clinical effects of liraglutide [15-20]. However, Yabe et al. reported that lixisenatide is well tolerated across all levels of $\beta$-cell function, highlighting the 
importance of the non-insulin-related effects of lixisenatide [22]. Clinical response prediction based on $\beta$-cell function-related indices would differ between liraglutide and lixisenatide.

\section{CONCLUSION}

Residual $\beta$-cell function, as assessed from the CPR-AUC via the GST, helps predict the efficacy of GLP-1ra. The once-a-week frequency of GLP1ras treatment is expected to increase owing to the low frequency of injection, safety for hypoglycemia, and expected cardiovascular effects. GST is a simple and easy clinically feasible approach. Accordingly, GST may help determine whether to switch to or add GLP-1ra treatment in patients with insulin-independent diabetes.

\section{ACKNOWLEDGEMENTS}

We thank all the study participants. We also appreciate the contributions of the nurses and doctors at the Department of Endocrinology and Metabolism of Kanazawa University Hospital.

Funding. No funding or sponsorship was received for this study or publication of this article. The article processing charges were funded by the authors.

Editorial Assistance. We thank Editage (Tokyo, Japan; www.editage.jp) for the English language editing.

Authorship. All named authors meet the International Committee of Medical Journal Editors (ICMJE) criteria for authorship for this article, take responsibility for the integrity of the work as a whole, and have given their approval for this version to be published.

Disclosures. Azusa Ohbatake, Kunimasa Yagi, Shigehiro Karashima, Yuki Shima, Hiroyuki Asaka, Yukiko Miyamoto, Satoko Okazaki, Mitsuhiro Kometani, Masa-aki Kawashiri, Yoshiyu Takeda, Takashi Yoneda, and Daisuke
Chujo declare that they have no conflict of interest.

Compliance with Ethics Guidelines. All procedures performed in studies involving human participants were approved by the ethics committee at the University of Kanazawa (no. 2014124), and were in accordance with the ethical standards of the national research committee and with the 1964 Helsinki Declaration and its later amendments or comparable ethical standards. This study used non-identifiable data obtained by the treating physicians and therefore, on the basis of the decision from our local ethics committee of University of Kanazawa (no. 2014124), informed consent was not required. Patients had the opportunity to object to the use of their data for retrospective scientific research; however, none of the patients did.

Data Availability. The data sets generated during and/or analyzed during the current study are available from the corresponding author on reasonable request.

Open Access. This article is distributed under the terms of the Creative Commons Attribution-NonCommercial 4.0 International License (http://creativecommons.org/licenses/ by-nc/4.0/), which permits any noncommercial use, distribution, and reproduction in any medium, provided you give appropriate credit to the original author(s) and the source, provide a link to the Creative Commons license, and indicate if changes were made.

\section{REFERENCES}

1. DeFronzo RA. The triumvirate: beta-cell, muscle, liver. A collusion responsible for NIDDM. Diabetes. 1988;37:667-87.

2. Stumvoll M, Goldstein BJ, van Haeften TW. Type 2 diabetes: principles of pathogenesis and therapy. Lancet. 2005;365:1333-46.

3. UK Prospective Diabetes Study (UKPDS) Group. Intensive blood-glucose control with sulphonylureas or insulin compared with conventional treatment and risk of complications in patients 
with type 2 diabetes (UKPDS 33). Lancet. 1998;352:837-53.

4. Funakoshi S, Fujimoto S, Hamasaki A, et al. Analysis of factors influencing pancreatic beta-cell function in Japanese patients with type 2 diabetes: association with body mass index and duration of diabetic exposure. Diabetes Res Clin Pract. 2008;82:353-8.

5. Funakoshi S, Fujimoto S, Hamasaki A, et al. Analysis of factors influencing postprandial C-peptide levels in Japanese patients with type 2 diabetes: comparison with C-peptide levels after glucagon load. J Diabetes Invest. 2012;2:429-34.

6. Drucker DJ. The cardiovascular biology of glucagonlike peptide-1. Cell Metab. 2016;24:15-30.

7. Cheang JY, Moyle PM. Glucagon-like peptide-1 (GLP-1)-based therapeutics: current status and future opportunities beyond type 2 diabetes. Chem Med Chem. 2018;13:662-71.

8. Marso SP, Daniels GH, Brown-Frandsen K, et al. Liraglutide and cardiovascular outcomes in type 2 diabetes. N Engl J Med. 2016;375:311-22.

9. Marso SP, Bain SC, Consoli A, et al. Semaglutide and cardiovascular outcomes in patients with type 2 diabetes. N Engl J Med. 2016;375:1834-44.

10. Asano T, Kawamura M, Watanabe T, et al. Indices of urinary and serum C-peptide corrected with fasting plasma glucose for decision-making of insulin therapy in type 2 diabetes-validation and comparison (in Japanese). J Jpn Diabetes Soc. 2008;51:759-63.

11. Funakoshi S, Fujimoto S, Hamasaki A, et al. Utility of indices using C-peptide levels for indication of insulin therapy to achieve good glycemic control in Japanese patients with type 2 diabetes. J Diabetes Investig. 2011;2:297-303.

12. Faber OK, Binder C. C-peptide response to glucagon. A test for the residual beta-cell function in diabetes mellitus. Diabetes. 1977;26:605-10.

13. Kondo Y, Satoh S, Osada UN, et al. Early liraglutide treatment improves $\beta$-cell function in patients with type 2 diabetes: a retrospective cohort study. Endocr J. 2015;62:971-80.
14. Jacobs ML, Verhoog S, van der Linden $\mathrm{WH}$, et al. Glucagon stimulation test: assessment of beta-cell function in gestational diabetes mellitus. Eur J Obstet Gynecol Reprod Biol. 1994;56:27-30.

15. Kondo Y, Satoh S, Nagakura J, et al. Defining criteria for the introduction of liraglutide using the glucagonstimulation test in patients with type 2 diabetes. J Diabetes Investig. 2013;4:571-5.

16. Usui R, Yabe D, Kuwata $\mathrm{H}$, et al. Retrospective analysis of safety and efficacy of insulin-to-liraglutide switch in Japanese type 2 diabetes: A caution against inappropriate use in patients with reduced $\beta$-cell function. J Diabetes Investig. 2013;4:585-94.

17. Usui R, Yabe D, Kuwata $\mathrm{H}$, et al. Retrospective analysis of safety and efficacy of liraglutide monotherapy and sulfonylurea-combination therapy in Japanese type 2 diabetes: association of remaining $\beta$-cell function and achievement of HbA1c target one year after initiation. J Diabetes Complications. 2015;29:1203-10.

18. Kozawa J, Inoue $\mathrm{K}$, Iwamoto $\mathrm{R}$, et al. Liraglutide is effective in type 2 diabetic patients with sustained endogenousinsulin-secreting capacity. J Diabetes Investig. 2012;3:294-7.

19. Kawata T, Kanamori A, Kubota A, et al. Is a switch from insulin therapy to liraglutide possible in Japanese type 2 diabetes mellitus patients? J Clin Med Res. 2014;6:138-44.

20. Iwao T, Sakai K, Sata M. Postprandial serum C-peptide is a useful parameter in the prediction of successful switching to liraglutide monotherapy from complex insulintherapy in Japanese patients with type 2 diabetes. J Diabetes Complications. 2013;27:87-91.

21. Yabe D, Seino Y. Defining the role of GLP-1 receptor agonists for individualized treatment of type 2 diabetes. Expert Rev Endocrinol Metab. 2014;9:659-70.

22. Yabe D, Ambos A, Cariou B. Efficacy of lixisenatide in patients with type 2 diabetes: a post hoc analysis of patients with diverse $\beta$-cell function in the GetGoal-M and GetGoal-S trials. J Diabetes Complications. 2016;30:1385-92. 